\title{
Discussion on How to Supervise the Safety of Temporary Electricity in Construction
}

\author{
Zhang Hua, Yang Xiaolei, Lan Chenghao, Wang Shuai, Liu Jia, Dong Pin, Chen Hui, Feng \\ Lei, Wang Qi, Hu Yang
}

Fushun Power Supply Company, Liaoning Electric Power Company Limited, State Grid, China

Keywords: Temporary electricity; Electrical safety; Safety

\begin{abstract}
In view of the safety problem of construction temporary electricity use, it is expounded that the distribution network has low voltage level, but the types of electrical equipment are complex, the users are uneven, and there is a great risk of electric shock. Aiming at the problem of leakage in distribution line, the system analysis of leakage protection is carried out first, and then the correct use of leakage protection in distribution network is studied. In the end, the common problems of leakage protection are discussed from the aspects of human and material, and the solutions are given. The research of leakage protection has the significance of promoting the application of leakage protection. It can let the staff understand the correct use of leakage protection, and has a certain promotion value.
\end{abstract}

\section{Introduction}

At present, for the safety problem of temporary electricity in construction, due to the lack of management of the construction enterprise, there is no special organization design plan for construction electricity, or the scheme has not been examined and verified by the supervision unit. The result is that the hidden trouble of temporary electricity in construction site is constant, and it is easy to cause heavy casualties. In view of this, this paper discusses how the staff of the construction quality and safety supervision station can effectively supervise the safety of temporary electricity in construction for many years, in order to looking forward to mutual promotion and common improvement of safety level.

\section{Common Problems with Temporary Electricity}

During the normal inspection process, the following seven common problems of temporary electricity in construction are often found:

1) Temporary electricity is up to 5 or total capacity reaches $50 \mathrm{~kW}$, and the construction organization is not designed for temporary electricity.

2) In the same power network, the protective zero line and the protective grounding are adopted simultaneously.

3) The protective zero lines are iterative grounding in less than three places (starting, middle, end), the grounding resistance of each grounding device is more than 10 ohms.

4) The leakage protector of the main distribution box does not reach the leakage action current value of $300 \mathrm{~mA}$ to $1000 \mathrm{~mA}$, the distribution box does not reach $100 \mathrm{~mA}$ to $200 \mathrm{~mA}$, the switch box is more than $30 \mathrm{~mA}$, the rated leakage action time is more than $0.1 \mathrm{~s}$.

5) Automatic air circuit breaker (short for air switch), which is used as isolating switch.

6) The wooden electric box and the wooden bottom plate of the metal shell are used, the distribution box is not marked with its name, use, and no lock.

7) the cross section of the protective zero line is greater than that of the working zero line. In view of these common problems, we have reworked out the technical disclosure of temporary electricity in construction. 


\section{Principles to be followed for temporary electricity}

The temporary electricity in construction must meet the requirements of Technical specification for installation of temporary electricity in construction(JGJ46-88). The following 22 aspects should be noted:

1)It is strictly prohibited for non-electrician workers to unlawfully pull and connect the wires. There must be at least one electrician present when moving the electric box and electrical equipment.

2) The metal case of all electrical equipment in the construction site must be connected to the special protective zero line.

3) The protective zero line should be laid separately and not for other purposes; the iterative earth connection should be connected to the protective zero line. The protective zero wire connected with electrical equipment shall be insulated multi-strand copper wire with a cross section of not less than $2.5 \mathrm{~mm}^{2}$. The uniform mark of protective zero line is green / yellow double color line. No green / yellow double color line is allowed to be used as load line under any circumstances.

4) Under normal conditions, the exposed conductive parts of the following uncharged electrical equipment shall be protective connection to zero. The metal case of electrical machinery, transformers, electrical appliances, lighting appliances, hand-held power tools; the metal casing for a transmission of electrical equipment; a metal shell for a distribution cabinet.

5) The exhaust pipe of the generator and diesel air compressor must be outstretched out of the air. Generators, diesel air compressors and their control and distribution rooms are strictly prohibited from storing oil barrels.

6) Overhead wire must be insulated copper wire or insulated aluminum wire.

7) Overhead lines must be set on special poles and are strictly forbidden to be assumed on trees and scaffolding.

8) The cable trunk line should be laid underground or overhead laying. It is strictly forbidden to set up along the ground, and mechanical damage and medium corrosion should be avoided.

9) When the rubber cable is laid overhead, it should be arranged along the wall or pole and fixed with insulators. It is strictly forbidden to use bare metal wire as binding wire. The distance between fixed points should ensure that the rubber cable can bear the load caused by deadweight. The maximum sag distance of rubber cable shall not be less than $2.5 \mathrm{~m}$.

10) Humid place or non-cable wiring buried in the ground must be piped laying, and the nozzle should be sealed. When the metal pipe is laid, it must be protective connection to zero.

11) The power distribution box and the lighting distribution box should be set separately. Once the power distribution box is installed in the same distribution box, the power and lighting lines should be separated.

12) Distribution box and switch box shall be installed correctly and securely. Mobile distribution box and switch box shall be installed on strong support.

13) The distribution box and the switch box must prevent rain and dust. The electric appliances in the box must be reliable and complete, and the damaged and unqualified electric appliances are not allowed.

14) Each electrical equipment must have its own special switchbox and must implement the "one machine, one gate" system. It is strictly prohibited to use the same switchgear to directly control two or more electrical equipment (including sockets).

15) The current leakage current of the leakage protector in the switch box should be less than 30 $\mathrm{mA}$, and the rated leakage time should be less than $0.1 \mathrm{~s}$. When a leakage switch is used in a wet place such as water pump, the rated operating current should not be greater than $15 \mathrm{~mA}$. The rated leakage time should be less than 0.1s.

16) Manual switchgear shall be used only for direct control of lighting circuits and power circuits of capacity not greater than $5.5 \mathrm{~kW}$. Power circuits with capacity greater than $5.5 \mathrm{~kW}$ shall be controlled by automatic switchgear or step-down starting device.

17) The inlet and outlet outlets of the conductor in the switchbox should be located on the bottom surface of the box, it is strictly prohibited to place them on the top, side, back or door of the 
box. The incoming and outgoing lines of mobile distribution box and switch box must use rubber insulated cable.

18) The power cord entering the switch box is strictly forbidden to adopt socket connection.

19) All distribution boxes should be named, used and marked as shunt.

20) All distribution box doors shall be locked, and the distribution box and switch box shall be handled by a specialist.

21) All distribution boxes and switchboxes shall be inspected and repaired once a month. Inspection and maintenance personnel must be professional electricians. Inspection and maintenance must be required to wear, wear insulating shoes, gloves, and must use electrical insulation tools.

22) When checking and repairing the distribution box and switch box, it is necessary to switch off the corresponding power supply switch of the former level, and the blackout sign should be hung, and the live working is strictly prohibited.

23) If the construction site stops working for more than one hour, the power switch box should be turned off and locked.

24) No sundries should be placed in the distribution box and switch box and should be kept clean and tidy at all times.

25) Other temporary electrical equipment cannot be attached to the distribution box and switchbox.

26) When the melt of the fuse is replaced, it is strictly prohibited to replace it with a melt that does not conform to the original specification. It is strictly prohibited to contact with the sharp fracture of metal and strong corrosive medium.

27) The inlet and outlet lines of the distribution box and switch box shall not be subjected to external force.

28) Iodized tungsten lamp is forbidden to be used in field lighting. Lighting device with rated voltage of $220 \mathrm{~V}$ is used in the field.

\section{The Content of Safety Supervision on Temporary Electricity}

The main contents of the work of temporary electricity safety supervision on construction site are as follows:

1) Checking whether the temporary electricity on construction site is accepted by the supervisor.

2) Checking whether the temporary electricity in construction site meets the requirements of Technical Specification for Safety of temporary electricity on Construction site (JGJ46-88).

3) Once the major safety hidden danger is discovered, the request of stopping work and rectification is put forward, and the relevant units are supervised to carry out the rectification.

\subsection{Drawing TN-S system}

According to the above three requirements, during the on-site inspection, we also carried an internally compiled schematic of the TN-S system:

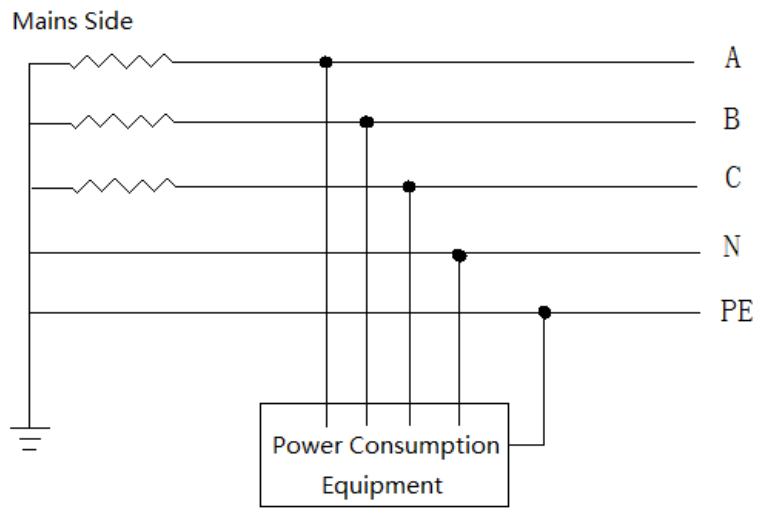

Fig.1 TN-S system wiring diagram 
TN-S system: three-phase five-wire system, it refers to five lines of $\mathrm{A}, \mathrm{B}, \mathrm{C}, \mathrm{N}$ and $\mathrm{PE}$, among which PE lines are protective earth wire, also known as safety lines. The utility model is specially used to ensure the safe use of electricity, such as the enclosure of the equipment. It is a power supply system that strictly separates the working zero-line $\mathrm{N}$ from the special protection line PE at the power supply source.

Through this TN-S system wiring diagram, and in addition to our on-site comments on physical objects, in a relatively short period of time, when the person in charge of the construction site, the full-time safety officer on the construction site and the person in charge of the electrician at the construction site have some questions about what is the TN-S system, what is the two-level protection of the three-level distribution system, and so on, it will give them a clear idea and also make our inspection more effective than expected.

\subsection{Using three-stage distribution two-stage protection}

Three-stage distribution two-stage protection: three-level distribution refers to three standard boxes, the distribution of the main distribution box, distribution box and switch box. The switch box shall conform to one machine, one box, one gate and one leak. All kinds of electrical appliances in the three types of electric boxes should be qualified products, and two-stage protection refers to two-stage protection refers to leakage protectors in general distribution boxes and switch boxes that meet capacity and quality requirements.

\subsection{Increase supervision of on-site supervisors}

It has increased supervision of on-site supervisors. First of all, in accordance with the relevant specifications, the director of the project department of the supervision unit is required to write in the supervision specifications prepared before the commencement of construction, as well as the supervision rules for examination and verification, and to include the safety inspection of temporary electricity in the construction, and should be supervised and implemented by a professional supervisory engineer. And repeatedly stress one point, according to the relevant laws and regulations. In the course of inspection, the supervision unit finds hidden dangers and requires the construction unit to rectify and rectify it. If the construction unit refuses to perform it, it has the right to report to the station. If the supervision unit fails to effectively urge the construction unit to rectify and reform in place, and fails to report to the relevant construction administrative department, the station will be in accordance with the corresponding laws, regulations to the supervision unit for serious treatment.

\section{Conclusion}

As a result of the temporary electricity on construction site, an additional safety supervisor has been added to each construction project. They are mainly responsible for supervising the problem of using electricity for construction, and organize manpower at any time to carry out random spot checks on the construction site. Therefore, through strict management of construction electricity in various aspects, it has effectively controlled the chaotic situation of the temporary electricity management in the construction site, and greatly reduced the safety problems of the construction site.

\section{References}

[1] Zhao Honghai. Discussion on improving the way of leakage protection in rural low voltage power grid [J]. 2002,19 (3): 132-135.

[2] $\mathrm{Li}$ Shibo. Analysis and solving measures of Daily failure of leakage protector[J]. communication world.2007.

[3] Zhao Shaofeng, Shi Yubo. On the safe operation and management of power dispatching [J], electronic technology, 2011 (5).

[4] Yang Mingfang, Wang Kunlun. Elementary introduction to the safety management of electric power operation [J], economic forum, 2011 (11). 